\title{
Interaction Between Sex and Cardiac Interoceptive Accuracy in Measures of Induced Pain
}

\author{
Eszter Ferentzi ${ }^{*}$, Mattis Geiger², Sandra A. Mai-Lippold ${ }^{3}$, Ferenc Köteles' \\ Christian Montag ${ }^{4}$ and Olga Pollatos ${ }^{3}$
}

${ }^{1}$ Institute of Health Promotion and Sport Sciences, ELTE Eötvös Loránd University, Budapest, Hungary, ${ }^{2}$ Department of Individual Differences and Psychological Assessment, Institute of Psychology and Education, Ulm University, Ulm, Germany, ${ }^{3}$ Department of Clinical and Health Psychology, Institute of Psychology and Education, Ulm University, UIm, Germany,

${ }^{4}$ Department of Molecular Psychology, Institute of Psychology and Education, Ulm University, Ulm, Germany

OPEN ACCESS

Edited by:

Anna Maria Aloisi,

University of Siena, Italy

Reviewed by:

Jun Yin,

Ningbo University, China

Vivien Ainley,

Royal Holloway, University of London, United Kingdom

*Correspondence: Eszter Ferentz

ferentzi.eszter@ppk.elte.hu

Specialty section:

This article was submitted to

Perception Science,

a section of the journal

Frontiers in Psychology

Received: 30 June 2020 Accepted: 31 December 2020

Published: 09 February 2021

Citation:

Ferentzi E, Geiger M,

Mai-Lippold SA, Köteles F, Montag C and Pollatos O (2021) Interaction

Between Sex and Cardiac Interoceptive Accuracy in Measures of Induced Pain.

Front. Psychol. 11:577961. doi: 10.3389/fpsyg.2020.577961
Pain perception is influenced by several factors, and among them, affect, sex, and perception of bodily signals are assumed to play a prominent role. The aim of the present study is to explore how sex, cardiac interoceptive accuracy, and the interaction of the latter two influence the perception of experimentally induced pain. We investigated a large sample of young adults $(n=159,50.9 \%$ female, age: $23.45, \mathrm{SD}=3.767$ ), assessing current positive and negative affective state with the Positive and Negative Affect Schedule (both involved as control variables), cardiac interoceptive accuracy with the mental heartbeat tracking task, and pain sensitivity with electrical stimulation on the back of the dominant hand, applying a repeated-measures staircase protocol. Males showed a significantly higher pain threshold and tolerance level than females, whereas cardiac interoceptive accuracy was not associated with pain sensitivity. The impact of sex $\times$ cardiac interoceptive accuracy interaction was significant for pain threshold only, while pain tolerance was predicted only by sex. According to these findings, the associations between pain sensitivity, cardiac IAc, and sex might be more complicated than it was supposed in previous studies. Interactions between factors impacting pain perception appear worthy of further investigation.

Keywords: interoception, cardiac interoceptive accuracy, pain perception, affect, sex differences

\section{INTRODUCTION}

Pain is defined as an unpleasant experience with sensory and emotional components, associated with potential tissue damage (Merskey and Bogduk, 1994), and strongly influenced by somatic, psychological, and social factors (Moseley, 2007). Neuroanatomical evidence demonstrates that pain processing shows a considerable overlap, with processing of visceral signals representing the actual homeostatic condition of the body; pain was also called the homeostatic emotion (Craig, 2003). Based on these findings, a broad perspective on interoception was proposed, which includes pain, itch, sensual touch, the sense of the metabolic state of the muscles, and other modalities beyond the classic visceroceptive channels (Craig, 2010). Concerning the conscious aspects of interoception, Garfinkel and colleagues (Garfinkel et al., 2015) proposed a multifaceted model, which differentiates objective (called interoceptive accuracy, IAc), subjective (interoceptive sensibility), and metacognitive (interoceptive awareness) dimensions of interoception. Although cardiac IAc cannot be generalized to other interoceptive modalities (Ferentzi et al., 2018), it is often 
used as an indicator of the acuity of perception of interoceptive signals. As the experience of pain is accompanied by a marked cardiovascular response (Gracely, 1999), the use of cardiac IAc is well justified in this area of research.

Both interoception and pain are interpreted as sensitivity towards bodily or body-related signals, associated with cognitive and emotional regulatory processes (Craig, 2003, 2015); thus, a connection between the two constructs seems plausible. However, the relationship of cardiac IAc and pain is not yet well understood, as empirical evidence provided so far is contradictory.

A systematic review drew the conclusion that patients with chronic pain syndromes are characterized by a less accurate perception of heartbeats than healthy control subjects (Di Lernia et al., 2016). Chronicity of the condition, however, might play an important role in these results. Studies that investigated non-clinical samples either found that more accurate heartbeat perception (as assessed by the mental heartbeat tracking task; Schandry, 1981) was associated with decreased pain threshold and tolerance (Pollatos et al., 2012; Weiss et al., 2014) or revealed no association (Werner et al., 2009; Ferentzi et al., 2018). It is an open question what might explain these contradictory findings. Pollatos et al. (2012) investigated 60 participants and found a correlation of medium effect size (cardiac IAc and pain threshold: $r=-0.42 ; p<0.01$; cardiac IAc and pain tolerance: $r=-0.33, p<0.05)$ similarly to Weiss et al. (2014; $n=30$, cardiac IAc and pain threshold: $r=-0.53$; $p<0.01$; cardiac IAc and pain tolerance: $r=-0.44, p<0.05$ ); both studies used a pressure algometry to induce pain. Both the studies of Weiss et al. (2014) and Werner et al. (2009) used preselected samples. The former investigated a clinical sample as well, while the latter matched samples of people with high and low heartbeat perception $(n=62)$. The two studies that found no association applied heat (Werner et al., 2009) or induced ischemic pain (Ferentzi et al., 2018). The diversity of the applied methodologies could partly influence the incoherence of the findings.

A candidate to help understanding the pain and cardiac IAc relationship is sex because it seems to relate to both pain perception and cardiac IAc. Males usually show higher pain threshold and tolerance level (Rhudy and Williams, 2005; Fillingim et al., 2009) as well as higher cardiac IAc (Whitehead et al., 1977; Jones and Hollandsworth, 1981; Katkin et al., 1981; Katkin, 1985; Harver et al., 1993; Montoya et al., 1993; Grabauskaite et al., 2017). Interestingly, there is no agreement concerning the explanation of the latter finding. Body composition might be an influencing factor (Montgomery et al., 1984; Jones et al., 1987) and could be in the background of sex differences in IAc (Rouse et al., 1988). There is a study investigating children, but it still found differences between males and females even after controlling for body mass index (Koch and Pollatos, 2014). Cardiodynamic characteristics (e.g., stroke volume and the momentum of ejected blood mass; Schandry et al., 1993; Khalsa et al., 2009) may also lie behind sex differences in heartbeat perception. Moreover, the existence of sex difference in cardiac IAc is not uniformly supported by empirical findings; there are studies that reported no divergence between sexes (e.g., Mussgay et al., 1999; Pollatos and Schandry, 2004).
Unlike cardiac IAc, the difference between males and females in pain sensitivity is widely supported by empirical studies (Fillingim et al., 2009). According to recent findings, females show higher pain sensitivity than males independently from the applied experimental pain evoking modality (Ostrom et al., 2017). Sex differences in pain perception have been explained by various modulating psychological factors (Ostrom et al., 2017); however, differences in cardiac IAc or other interoceptive modalities have not been mentioned among these. In summary, males are characterized by higher cardioceptive IAc and lower pain sensitivity than females. This might indicate that sex plays a moderating role on the association between interoception and pain sensitivity.

Furthermore, according to theoretical accounts and empirical investigations, both pain perception and cardiac IAc are tightly linked to affect. In the case of pain, it is not clear which aspect of affect is relevant. On the one hand, most studies investigating the effect of experimentally induced emotions found that pleasant affective states are associated with higher pain threshold and tolerance, and negative states with increased sensitivity toward pain (Meagher et al., 2001; Carter et al., 2002; Villemure et al., 2003; Rainville et al., 2005). According to other findings, on the other hand, the emotion stimulation itself (along with further modifying factors, such as attention) is associated with reduced pain sensitivity, while the emotional valence of the stimulation is less relevant (Arntz et al., 1991; Arntz and de Jong, 1993; Villemure, 2002; de Tommaso et al., 2009). Interoception, in general, is believed to be tightly linked to emotional experience (James, 1884; Cannon, 1927; Damasio, 2010). Recent empirical studies applying emotional stimuli seem to confirm this view (e.g., Herbert et al., 2007, 2010; Pollatos et al., 2007a). There is evidence that the spontaneous affective state is also related to interoception. For example, state anxiety is associated with higher cardiac IAc as assessed by mental heartbeat tracking task (Schandry, 1981; Ludwick-Rosenthal and Neufeld, 1985; Pollatos et al., 2007b). Thus, controlling for the affective state might shed more light on the relationships between sensitivity to pain, cardiac IAc, and sex.

The aim of the present study was to better understand how current affective state, heartbeat perception ability, and sex influence pain perception in healthy individuals. Our hypotheses are that higher cardiac IAc is associated with lower (1) pain threshold and (2) pain tolerance. Furthermore, sex differences with respect to pain sensitivity were also assumed; we expected (3) higher pain threshold and (4) tolerance for males than females and (5) an interaction between sex and cardiac IAc on measures of pain.

\section{MATERIALS AND METHODS}

\section{Participants}

A total of 159 university students (50.9\% female, mean age: 23.45, $\mathrm{SD}=3.767)$ participated in the study. They were compensated with 20€. The exclusion criteria included psychiatric or neurological illnesses with current, not treated symptoms. The participants were fluent in German and were recruited from 
a local database called Ulm Gene Brain Behavior Project. Participation was voluntary and anonymous. All participants signed a written informed consent before the start of the measurements. Ethical approval for the study was obtained from the ethics committee of Ulm University, Ulm, Germany.

As data collection was part of a larger study (see the details below), a priori sample size calculation was not possible. However, the minimum required sample size for linear multiple regression with five tested predictors and medium effect size $\left(f^{2}=0.15\right)$ is $n=138(\alpha=0.05$, power $=0.95$; calculated with $G^{*}$ Power v3.1.9.4. software, Faul et al., 2007). As the sample size of the present study exceeds this threshold, it can be considered appropriate for testing the hypotheses introduced above. We assumed medium effect size based on the study of Pollatos et al. (2012); this study investigated a similar sample and reported a correlation of $r=-0.42(p<0.01)$ between IAc and pain threshold and $r=-0.33(p<0.05)$ between cardiac IAc and pain tolerance.

\section{Study Protocol}

The data collection started with the Positive and Negative Affect Schedule (PANAS) questionnaire (Watson et al., 1988). After that, all the electrodes were placed (measuring electrocardiogram, electromyogram, and skin conductance-the data of the latter two are not used here) using a portable BIOPAC system, Biopac MP150 system with the modules UIM100C, STP100C, ECG100C, GSR100C, and two EMG100C modules. Data were acquired with AcqKnowledge 4.1.1 (BUILD: 04.23.2010; 07.14.2010c; DISK46). This was followed by the heartbeat tracking task and the pain perception task. The data collection occurred as part of a larger study. The results of the measurements that followed are not reported here (i.e., pain regulation task, cognitive tasks, and emotion expression task).

\section{Measures}

\section{Positive and Negative Affect}

The PANAS aims to assess positive (PA) and negative (NA) emotional states (Watson et al., 1988). We used the 20-itemlong version of the PANAS that measures the current affective state of the participants, with 10 items per each affect (Watson et al., 1988). High scores refer to high level of affect. The internal consistencies assessed by Cronbach's alpha were $\alpha=0.84$ (PA) and 0.83 (NA).

\section{Cardiac Interoceptive Accuracy}

Cardiac IAc was measured with the mental tracking method (Schandry, 1981) over four different periods of time with various length $(25,35,45$, and $60 \mathrm{~s})$ presented in random order. The participants were seated comfortably, with palms facing up on a table in front of them. They were asked to count their heartbeats silently while not using any extra tricks to feel their heartbeats better. The participants were encouraged to report any number of heartbeats they could feel by emphasizing that there are no right and wrong answers and by adding that there are not many people who can feel their heartbeats correctly. If they were uncertain or could not feel their heartbeats, they were encouraged to still try to count or estimate the number of heartbeats. The accuracy score was calculated for each trial using the following formula: 1 - | (recorded heartbeats-counted heartbeats)/recorded heartbeats|; the measure of cardiac accuracy score was then calculated as an aggregate mean score over all items, ranging between 0 and 1 . Thus, higher values indicate better IAc. Cronbach's $\alpha$ for the four trials is 0.95 in the current study.

\section{Pain Perception}

Pain-related perception was measured with a psychophysical upward staircase threshold estimation task characterized by two thresholds: the pain threshold (when the slightest pain, i.e., more than an unpleasant sensation was felt for the first time) and the pain tolerance (when pain was not tolerable anymore). Pain stimuli were applied via electrical stimulation on the back of the dominant hand; the cathode was applied to the middle phalanx of the index and the anode to the proximal phalanx of the second finger $(400 \mathrm{~V}$; with a starting point of $0.1 \mathrm{~mA}$, increasing by $0.1 \mathrm{~mA}$ with each trial to set the sensation threshold first and by $0.5-\mathrm{mA}$ steps to measure pain threshold and tolerance). The length of each trial was $5 \mathrm{~s}$ of stimulation, with a 6-10-s-long break in between.

The participants were instructed that the aim of the study is to assess their sensitivity to electrical impulses. They were also informed that they will feel pain at a certain point of the assessment. In the case of pain threshold, they were asked to report when they feel the smallest signs or sensations of pain; in case of pain tolerance, they were asked to report when they cannot bear the pain anymore and want to interrupt the stimulation.

The participants verbally indicated when a stimulus crossed the currently assessed threshold. The stimulus intensity (in $\mathrm{mA}$ ) rated as crossing the threshold (first painful or maximally tolerable stimulus) was taken as a threshold value. Stimulation was immediately interrupted if the participants reported that it crossed the tolerance threshold.

Pain threshold was assessed three times (average correlation between the three values is rho $=0.955, p<0.001)$. The assessment re-started at the lowest threshold, minus three steps $(0.3$ or $1.5 \mathrm{~mA})$. Pain tolerance was assessed two times (rho $=0.975, p<0.001$ ). The final score for each threshold was the average of the respective trials.

\section{Data Analyses}

Statistical analysis was performed with JASP software 0.9.1 (JASP Team, 2019). According to the Shapiro-Wilk test of normality, only PA was normally distributed ( $p=0.354$; for all the other variables, $p<0.001$ ). Therefore, Spearman correlations were computed to assess the associations between the variables. As sex has a strong influence on subjective pain ratings, sex differences were investigated separately with Mann-Whitney $U$-test. Effect sizes (ES) were estimated as a rank-biserial correlation. As the frequentist approach to statistical hypothesis testing has recently received criticism (Dienes, 2011; Jarosz and Wiley, 2014), hypotheses were tested using both frequentist and Bayesian methods.

Based on visual inspection, five outlier values were identified for both pain perception values at the trial level (from two participants); these values were winsorized, i.e., the 
original values were replaced by the nearest value from the same variable set.

To analyze the effect of positive and negative affective state, heartbeat perception score, and sex on pain perception, both frequentist and Bayesian regression analyses were conducted. In the frequentist approach, two different linear regression analyses were conducted for pain threshold and pain tolerance. The variables were entered using the ENTER method in four steps: (1) positive and negative affect, (2) sex (males $=1$, females $=2$ ), (3) heartbeat perception score, and (4) sex $\times$ heartbeat perception score interaction term. The interaction term was calculated as the product of the centered values of sex and heartbeat perception scores.

In the Bayesian approach, the null model always included positive and negative affect (see the details below). The results are presented as Bayes factors $\left(\mathrm{BF}_{10}\right)$, showing the likelihood of the alternative hypothesis as compared to the null hypothesis. $\mathrm{BF}_{10}$ between 1 and 3 represent anecdotal, between 3 and 10 represent substantial, between 10 and 30 represent strong, between 30 and 100 represent very strong, and $>100$ represent decisive evidence in favor of the alternative hypothesis (Jeffreys, 1998; Jarosz and Wiley, 2014).

\section{RESULTS}

\section{Descriptive Statistics and Correlation Analyses}

Table 1 presents the descriptive statistics and the correlations of the measured variables, while Table 2 presents the findings of the Bayesian correlation analysis. In summary, the results of both frequentist and Bayesian analyses indicate a weak positive correlation between positive affect and pain tolerance. The frequentist approach also shows a weak association between positive affect and pain threshold, whereas associations between pain sensitivity and negative affect were not supported. In fact, $\mathrm{BF}_{10}$ values indicated the superiority of the null hypothesis (i.e., the lack of association with positive and negative affect) for both tolerance and threshold. There is also a weak positive correlation between pain tolerance and cardiac IAc according to the frequentist analysis.

\section{Sex Differences in Pain Measures}

Significant sex differences with respect to both assessed painrelated variables were found. The males showed significantly higher pain threshold ( $W=4,342, p<0.001, \mathrm{~d}=0.374$ ) and pain tolerance level $(W=5,401, p<0.001, d=0.710)$ than the females. The Bayesian analysis indicated decisive evidence both for pain threshold and tolerance $\left(\mathrm{BF}_{10}=123\right.$ and $\mathrm{BF}_{10}=69511$, respectively) in favor of the alternative hypotheses.

\section{Interaction Between Sex and Cardiac IAc on Pain Threshold and Pain Tolerance}

The final equation of the linear regression analysis with pain threshold as criterion variable was significant $[F(5,153)=6.214$, $p<0.001, R^{2}=0.169$ ], with sex $(\beta=-0.324, p<0.001$; males showing higher values) and sex $\times$ cardiac IAc interaction $(\beta=-0.192, p=0.014)$ as significant predictors (see details in Table 3). Concerning the interaction, higher levels of heartbeat tracking ability were associated with disproportionately lower levels of pain threshold in females compared to males. Figure 1 shows how cardiac IAc relates to pain threshold by gender. The ghost line on the right shows the fitted regression line made for males. Note that the fitted lines of the figure do not consider the impact of positive and negative affect.

The final equation predicting pain tolerance was also significant $\left[F(5,153)=18.354, p<0.001, R^{2}=0.375\right]$. The males were again characterized by higher values $(\beta=-0.519$, $p<0.001)$. The interaction term was just above the accepted level of statistical significance $(\beta=-0.126, p=0.061)$, just like the cardiac IAc scores $(\beta=0.120, p=0.067$; Table 4). Figure 2 shows how cardiac IAc relates to pain threshold by gender. (Similar to Figure 1, the ghost line on the right shows the fitted regression line made for males. Note also that the fitted lines of the figure do not consider the impact of positive and negative affect. The cardiac IAc and indicators of the current affective state were not associated with indicators of pain sensitivity.

In the Bayesian approach, when the null model included positive and negative affect and the alternative model included sex, the Bayes factor was $\mathrm{BF}_{10}=539.991$ for pain threshold and $\mathrm{BF}_{10} 1.075 \mathrm{e}+10$ for pain tolerance (which means that there is decisive evidence in favor of the alternative model). When sex was also added to the null model and the alternative model contained cardiac IAc, $\mathrm{BF}_{10}=0.567$ for pain threshold and $\mathrm{BF}_{10}=0.912$ for pain tolerance. In other words, the analyses were inconclusive with respect to the superiority of the null and the alternative model. The regression model predicting pain threshold that included sex $\times$ heartbeat perception score interaction term was almost five times more likely $\left(\mathrm{BF}_{10}=4.695\right.$, indicating substantial evidence in favor of the alternative model) as the null model (including positive and negative affect, sex, and heartbeat perception score). In the case of pain tolerance, however, the alternative model was not more likely to occur $\left(\mathrm{BF}_{10}=1.092\right)$. Thus, the Bayesian regression analyses supported the results of the frequentist analyses regarding the interaction term.

\section{DISCUSSION}

This study investigated the associations between sex and cardiac interoceptive accuracy with measures related to pain perception, i.e., pain threshold and pain tolerance, in 159 young individuals. After controlling for negative and positive affective state, the interaction between sex and cardiac IAc was a significant predictor of pain threshold, but not for pain tolerance.

The current affective state shows no consistent associations with pain sensitivity, indicating that the natural levels of positive and negative affect do not influence the sensitivity to experimentally induced pain in young individuals. The correlation between positive affect and pain tolerance, however, partly supports the view mentioned in the introduction, namely, that pleasant affective states are associated with lower pain sensitivity (Meagher et al., 2001; Carter et al., 
TABLE 1 | Descriptive statistics (mean, standard deviation and minimum-maximum values in the diagonal list) of the assessed variables and Spearman correlation coefficients (with rho and $p$ values); the last two columns present the data divided by sex.

\begin{tabular}{|c|c|c|c|c|c|c|c|}
\hline$N=159$ & PA & NA & $\begin{array}{l}\text { Cardiac } \\
\text { IAc }\end{array}$ & pain th. & pain tol. & Male, $N=78$ & $\begin{array}{c}\text { Female, } \\
N=81\end{array}$ \\
\hline PA & $\begin{array}{l}31.51 \pm 5.808 \\
\quad(17-47)\end{array}$ & $\begin{array}{c}0.010 \\
(0.903)\end{array}$ & $\begin{array}{c}0.112 \\
(0.158)\end{array}$ & $\begin{array}{c}0.162 \\
(0.042)\end{array}$ & $\begin{array}{c}0.244 \\
(0.002)\end{array}$ & $\begin{array}{c}32.94 \\
\pm 5.957 \\
(17-47)\end{array}$ & $\begin{array}{c}30.14 \\
\pm 5.344 \\
(19-46)\end{array}$ \\
\hline NA & & $\begin{array}{c}13.21 \pm 3.845 \\
(10-35)\end{array}$ & $\begin{array}{l}0.002 \\
(0.979)\end{array}$ & $\begin{array}{l}-0.064 \\
(0.421)\end{array}$ & $\begin{array}{l}-0.053 \\
(0.511)\end{array}$ & $\begin{array}{c}12.91 \\
\pm 3.358 \\
(10-26)\end{array}$ & $\begin{array}{c}13.50 \\
\pm 4.262 \\
(10-35)\end{array}$ \\
\hline pain th. & & & & $\begin{array}{l}2.31 \pm 1.455 \\
(0.50-6.50)\end{array}$ & $\begin{array}{c}0.641 \\
(<0.001)\end{array}$ & $\begin{array}{c}2.82 \\
\pm 1.626 \\
(0.50-6.50)\end{array}$ & $\begin{array}{c}1.82 \\
\pm 1.070 \\
(0.50-5.33)\end{array}$ \\
\hline pain tol. & & & & & $\begin{array}{c}6.03 \pm 3.174 \\
(1.00-17.25)\end{array}$ & $\begin{array}{c}7.86 \\
\pm 3.343 \\
(1.00-17.25)\end{array}$ & $\begin{array}{c}4.27 \\
\pm 1.651 \\
(1.50-8.50)\end{array}$ \\
\hline
\end{tabular}

Two-tailed in all cases.

PA, positive affect; NA, negative affect; IAc, interoceptive accuracy; pain th., pain threshold (in mA); pain tol., pain tolerance (in mA).

TABLE 2 | Results of the Bayesian correlation analysis (Kendall's tau-b coefficients).

\begin{tabular}{lcccc}
\hline $\boldsymbol{N}=\mathbf{1 5 9}$ & Negative affect & Heartbeat perception score & Pain threshold (mA) & Pain tolerance $(\mathbf{m A})$ \\
\hline Positive affect & 0.007 & 0.083 & 0.117 & -0.043 \\
Negative affect & & -0.003 & 0.072 & -0.036 \\
Heartbeat perception score & & & 0.132 & $0.485^{\star * *}$ \\
Pain threshold $(\mathrm{mA})$ & & & & \\
\hline
\end{tabular}

${ }^{*} B F_{10}>10 ;{ }^{* *} B F_{10}>100$.

TABLE 3 | Results of multiple linear regression analysis with pain threshold as criterion variable.

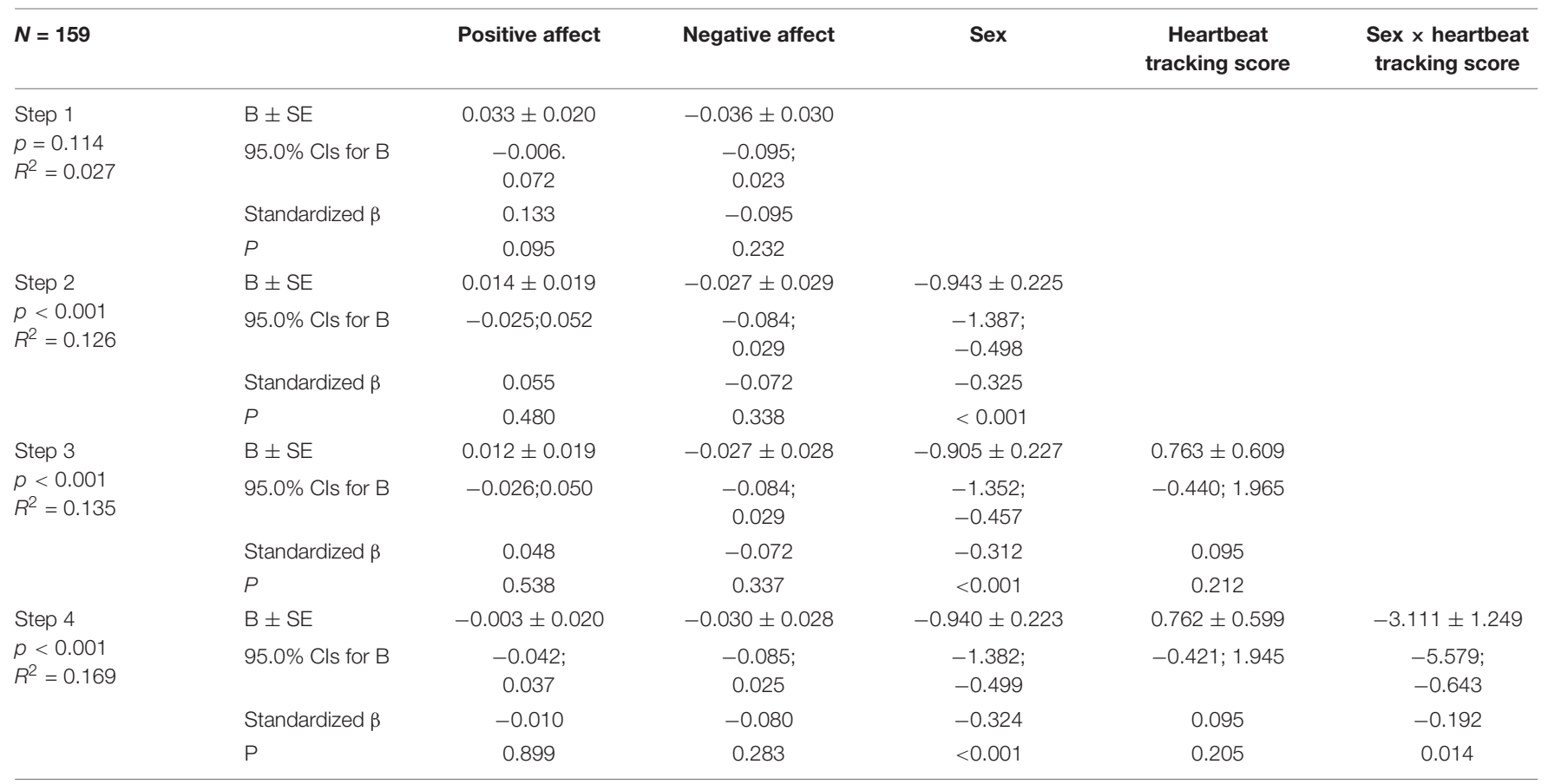

$B$, unstandardized beta; $S E=$ standard error for the unstandardized beta. 


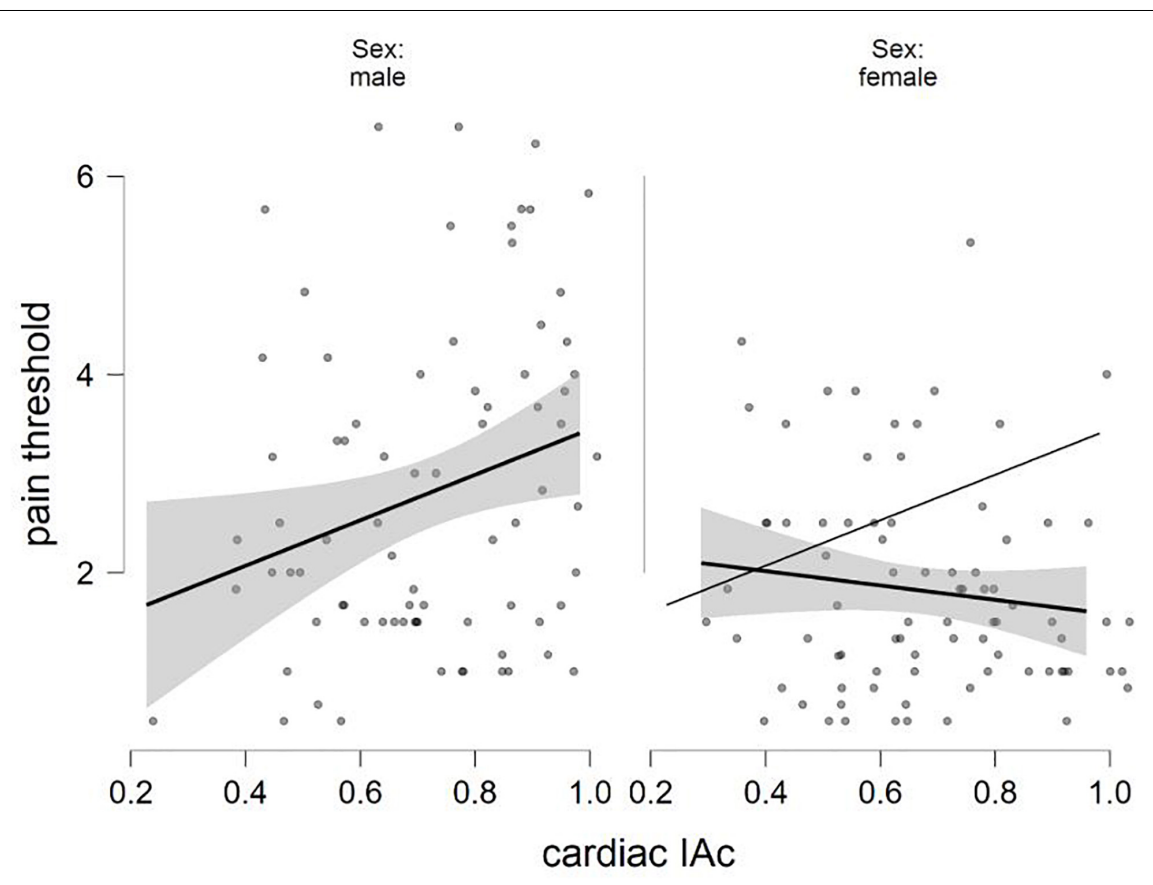

FIGURE 1 | Negative interaction between sex and cardiac IAc in the prediction of pain threshold (fitted regression lines with confidence intervals).

TABLE 4 | Results of multiple linear regression analysis with pain tolerance as criterion variable.

\begin{tabular}{|c|c|c|c|c|c|c|}
\hline$N=159$ & & Positive affect & Negative affect & Sex & $\begin{array}{c}\text { Heartbeat } \\
\text { tracking score }\end{array}$ & $\begin{array}{c}\text { Sex } \times \text { heartbeat } \\
\text { tracking score }\end{array}$ \\
\hline \multirow{3}{*}{$\begin{array}{l}\text { Step } 1 \\
p<0.001 \\
R^{2}=0.086\end{array}$} & $\mathrm{~B} \pm \mathrm{SE}$ & $0.150 \pm 0.042$ & $-0.076 \pm 0.063$ & & & \\
\hline & Standardized $\beta$ & 0.274 & -0.092 & & & \\
\hline & $P$ & $<0.001$ & 0.230 & & & \\
\hline $\begin{array}{l}p<0.001 \\
R^{2}=0.346\end{array}$ & $95.0 \%$ Cls for B & $\begin{array}{l}0.009 \\
0.153\end{array}$ & $\begin{array}{l}-0.152 \\
0.060\end{array}$ & $\begin{array}{l}-4.178 \\
-2.501\end{array}$ & & \\
\hline & Standardized $\beta$ & 0.148 & -0.056 & -0.528 & & \\
\hline & $P$ & 0.029 & 0.391 & $<0.001$ & & \\
\hline \multirow{2}{*}{$\begin{array}{l}\text { Step 3 } \\
p<0.001 \\
R^{2}=0.360\end{array}$} & $\mathrm{~B} \pm \mathrm{SE}$ & $0.076 \pm 0.036$ & $-0.046 \pm 0.053$ & $-3.235 \pm 0.425$ & $2.093 \pm 1.142$ & \\
\hline & $95.0 \%$ Cls for B & $\begin{array}{l}0.004 \\
0.148\end{array}$ & $\begin{array}{c}-0.152 \\
0.059\end{array}$ & $\begin{array}{l}-4.075 \\
-2.395\end{array}$ & $-0.162 ; 4.349$ & \\
\hline \multirow{4}{*}{$\begin{array}{l}\text { Step } 4 \\
p<0.001 \\
R^{2}=0.375\end{array}$} & $\mathrm{~B} \pm \mathrm{SE}$ & $0.055 \pm 0.038$ & $-0.050 \pm 0.053$ & $-3.286 \pm 0.423$ & $2.092 \pm 1.132$ & $-4.459 \pm 2.363$ \\
\hline & 95.0\% Cls for B & $\begin{array}{l}-0.019 \\
0.130\end{array}$ & $\begin{array}{c}-0.155 \\
0.054\end{array}$ & $\begin{array}{l}-4.121 \\
-2.451\end{array}$ & $\begin{array}{c}-0.145 \\
4.329\end{array}$ & $\begin{array}{l}-9.124 \\
0.212\end{array}$ \\
\hline & Standardized $\beta$ & 0.101 & -0.061 & -0.519 & 0.120 & -0.126 \\
\hline & $p$ & 0.145 & 0.345 & $<0.001$ & 0.067 & 0.061 \\
\hline
\end{tabular}

B, unstandardized beta; SE, standard error for the unstandardized beta.

2002; Villemure et al., 2003; Rainville et al., 2005). On the other hand, negative affect did not relate to any of the pain-related measures. In our setting, we focused only on the natural level of affect in a sample of healthy young adults, where negative affect was not particularly high and with a relatively low standard deviation as compared to positive affect.

Furthermore, it must be noted that the assessment of the natural level of affect with the PANAS might be considered as a measurement of personality traits; namely, that negative 


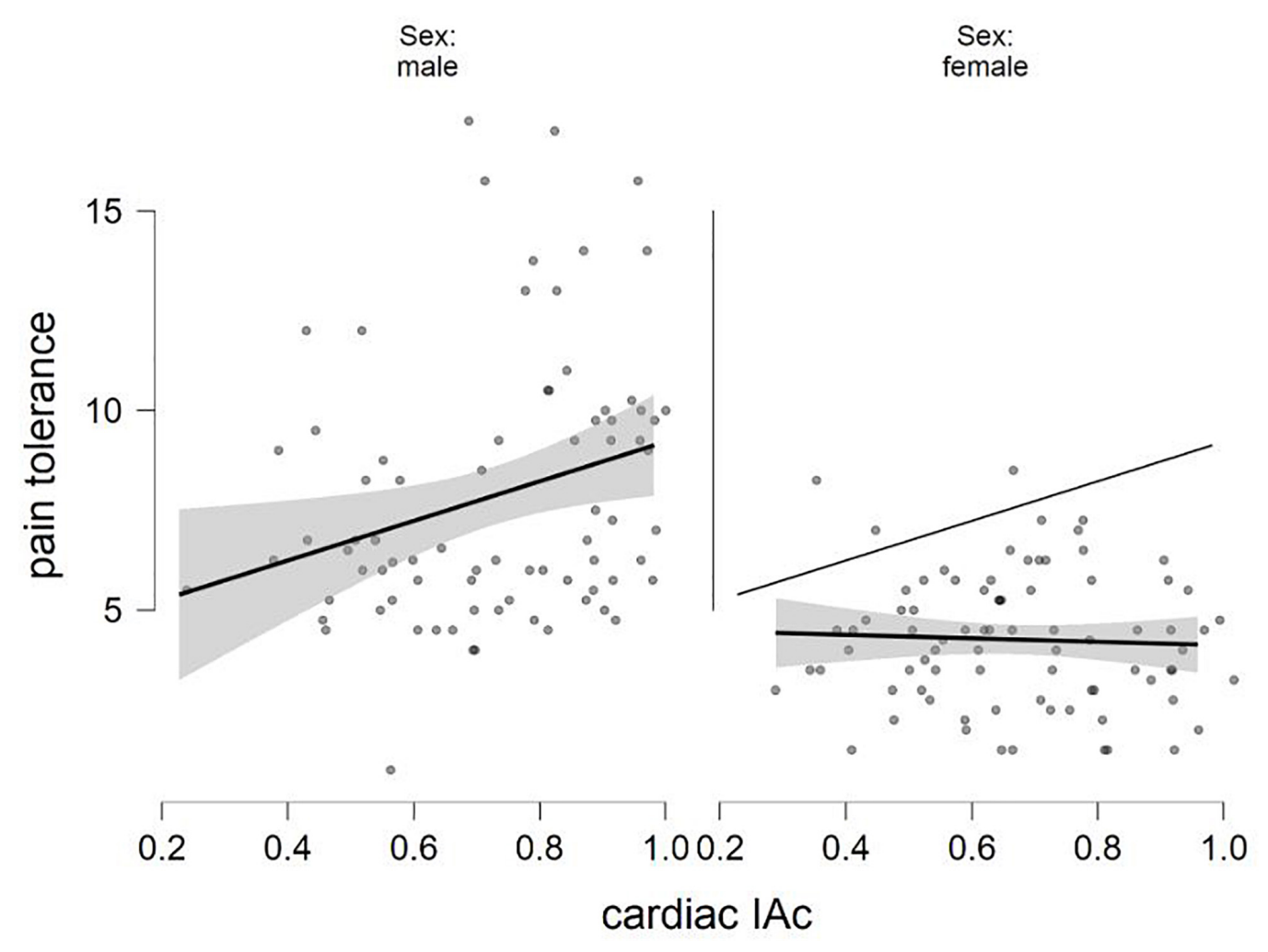

FIGURE 2 | The relation of sex and cardiac IAc in the prediction of pain tolerance (fitted regression lines with confidence intervals).

affect reflects the personality trait neuroticism and that positive affect reflects extraversion (Watson et al., 1992; for a review on state, personality traits, and personality change, see, e.g., Roberts, 2009). Thus, the relation of positive affect and pain sensitivity might reflect the well-documented extraversion pain sensitivity link (e.g., Lynn and Eysenck, 1961; Haslam, 1967).

The investigation of the relationship between pain, stress, and negative affect has a long tradition in pain research, with a strong focus on chronic pain conditions and negative affective states, such as depression and anxiety (Zautra et al., 2005). Studies applying various types of emotion evoking stimuli in healthy subjects (Meagher et al., 2001; Logan et al., 2003) were also not informative regarding the connection between pain perception and the natural changes of affect in the normal population. Our results show that, in a normal sample of young adults, the natural level of affect does not predict pain sensitivity.

Although the level of pain tolerance and threshold showed no association with cardiac IAc (hypotheses 1 and 2), we found interactions between sensitivity to pain and sex, i.e., males with higher level of interoceptive accuracy tend to have a higher pain threshold (hypothesis 5). These finding may be interpreted in the way that higher cardiac IAc (directly or indirectly) helps male participants to down-regulate their response to pain stimuli and thereby achieve higher pain threshold levels, whereas men with low cardiac IAc might rely more on response caution and report lower thresholds. The possible causes of this interaction will be discussed in more detail below.
Cardioceptive accuracy was not significantly associated with pain threshold. In fact, Bayesian correlation analysis indicated the superiority of the null hypothesis $(\mathrm{BF}<0.1)$. Concerning cardiac IAc and tolerance (hypotheses 2), a weak association was revealed in the frequentist correlation analysis, which was not supported by the Bayesian analysis. The association also became non-significant after controlling for sex and affective state in the regression analysis. This lack of association between the two modalities (i.e., pain sensitivity and heartbeat perception scores) that are both considered interoceptive by some authors based on their common neurological pathways (Craig, 2015) is in accordance with the results of previous studies reporting the independence of pain perception and heartbeat perception (Werner et al., 2009; Ferentzi et al., 2017, 2018) and suggesting that interoceptive ability, as it is assessed by one indicator only, is not necessarily generalizable to other internal sensory channels (Ferentzi et al., 2017, 2018). There are some additional factors, however, to take into account. Recent findings confirmed that pain sensitivity might have a modality-specific characteristic, describing distinct pain pathways of visceral and somatic pain (Hockley et al., 2017). Additionally, experimentally induced pain might have different characteristics depending on the method applied to evoke it (e.g. Goodin et al., 2012). Accordingly, the possibility that some types of internal sensory channels are linked not only at the neurophysiological level (Craig, 2015) but also at the level of the individual performance on the sensory measures cannot be excluded. For future studies, more careful 
consideration is needed to explore which types of sensory tests might be more likely to be linked together.

Both pain threshold and tolerance were predicted by sex. Male participants showed considerably higher pain threshold and tolerance level than females (hypotheses 3 and 4). The theoretically expected interaction between sex and heartbeat perception was also supported by the data for pain threshold (hypothesis 5). In summary, the major determinants of pain sensitivity in healthy individuals appear to be sex and to a lesser extent the interaction between cardiac IAc and sex.

According to our findings, the associations between pain sensitivity, cardiac IAc, and sex might be more complicated than it was supposed in previous studies. Whereas women show no association between indicators of pain sensitivity and cardiac IAc, cardiac IAc and pain threshold are inversely related in men (i.e., those with a higher level of cardiac IAc show a higher pain threshold). These results could be explained by the idea of Pennebaker (Pennebaker and Roberts, 1992). He explained the better performance of males at interoceptive tasks in laboratory circumstances by their better abilities to detect internal signals. Women, on the other hand, take into consideration more external (environmental) cues when estimating their current inner state. As both ways are reliable under everyday conditions, the sex difference is not necessarily apparent outside the laboratory where external cues are not controlled for Pennebaker and Roberts (1992). In future studies, it would be worthwhile to investigate the mechanisms of interoceptive abilities in everyday situations.

Males usually show a higher pain threshold and tolerance level (Rhudy and Williams, 2005; Fillingim et al., 2009) as well as higher cardiac IAc (Katkin, 1985; Montoya et al., 1993; Grabauskaite et al., 2017) (although, in the latter case, the findings are also controversial; Pennebaker, 1982; Mussgay et al., 1999; Pollatos and Schandry, 2004). Our results provide a more complete picture of the connection between the investigated variables. The association between higher pain threshold levels and higher cardiac IAc among men indicates that a sex-dependent extra mechanism might be involved. As pain can be characterized both by affective and by sensory components, it is possible that these can be regulated more effectively with higher inner focus, i.e., higher cardiac IAc among men. According to a brain imaging study, expectancies influence pain sensation (Atlas et al., 2010). A possible explanation of our result is that men with better cardiac IAc have higher expectancies concerning the pain stimulus, which makes them more prepared to get the pain stimulation that consequently becomes more manageable. A similar suggestion has been made by previous studies investigating interoception and emotion regulation (Füstös et al., 2013).

The current study has several limitations. First of all, the validity of the mental heartbeat tracking task by Schandry (1981) has been questioned by numerous authors (Ring et al., 2015; Desmedt et al., 2018; Murphy et al., 2018; Ring and Brener, 2018), and there is an ongoing debate about its validity (Zamariola et al., 2018; Ainley et al., 2020; Corneille et al., 2020; Zimprich et al., 2020). According to a recent study, the instructions allowing estimation could lead to different results than a stricter version of the instructions (Desmedt et al., 2020). However, in the original protocol of Schandry (1981), estimation was also allowed. The rationale of this is that heartbeats are weak sensations, and as such (similarly to ambiguous external sensations), estimation can reflect indeed a true but ambiguous sensation. Nevertheless, in the light of the recent findings and the debate mentioned above, more investigation is needed on the exact influence of the type instruction given.

Additionally, as pain has been stimulated electrically, it might not be generalizable to pain sensitivity induced differently, as it has been mentioned above. For example, a comparison of the experimental pain stimulation induced by four types of laser showed differences between the stimulation types (Svensson et al., 1991). The characteristics of the investigated sample (e.g., healthy young individuals) also limit the generalizability of the findings. Finally, the conclusion introduced above is valid only if the assumed medium effect size holds.

\section{DATA AVAILABILITY STATEMENT}

The raw data supporting the conclusions of this article will be made available by the authors, without undue reservation.

\section{ETHICS STATEMENT}

The studies involving human participants were reviewed and approved by Ethics Committee of Ulm University. The patients/participants provided their written informed consent to participate in this study.

\section{AUTHOR CONTRIBUTIONS}

MG, SAM-L, CM, and OP contributed to the conception and design of the study. MG contributed to the assessment of data, while EF and FK processed the data and performed the statistical analyses. EF wrote the first draft of the manuscript. MG, FK, and OP wrote sections of the manuscript. All authors read and commented on the final version of the manuscript.

\section{FUNDING}

EF was supported by a doctorate research grant funded by the German Academic Exchange Service (DAAD). 


\section{REFERENCES}

Ainley, V., Tsakiris, M., Pollatos, O., Schulz, A., and Herbert, B. M. (2020). Comment on "Zamariola et al. (2018), Interoceptive accuracy scores are problematic: evidence from simple bivariate correlations"-the empirical data base, the conceptual reasoning and the analysis behind this statement are misconceived and do not support the authors' conclusions. Biol. Psychol. 152:107870. doi: 10.1016/j.biopsycho.2020.107870

Arntz, A., and de Jong, P. (1993). Anxiety, attention and pain. J. Psychosom. Res. $37,423-431$.

Arntz, A., Dreessen, L., and Merckelbach, H. (1991). Attention, not anxiety, influences pain. Behav. Res. Ther. 29, 41-50.

Atlas, L. Y., Bolger, N., Lindquist, M. A., and Wager, T. D. (2010). Brain mediators of predictive cue effects on perceived pain. J. Neurosci. 30, 12964-12977. doi: 10.1523/JNEUROSCI.0057-10.2010

Cannon, W. B. (1927). The James-Lange theory of emotions: a critical examination and an alternative theory. Am. J. Psychol. 39, 106-124. doi: 10.2307/1415404

Carter, L. E., McNeil, D. W., Vowles, K. E., Sorrell, J. T., Turk, C. L., Ries, B. J., et al. (2002). Effects of emotion on pain reports, tolerance and physiology. Pain Res. Manag. 7, 21-30.

Corneille, O., Desmedt, O., Zamariola, G., Luminet, O., and Maurage, P. (2020). A heartfelt response to Zimprich et al. (2020), and Ainley et al. (2020)'s commentaries: acknowledging issues with the HCT would benefit interoception research. Biol. Psychol. 152:107869. doi: 10.1016/j.biopsycho.2020.107869

Craig, A. D. (2003). A new view of pain as a homeostatic emotion. Trends Neurosci. $26,303-307$

Craig, A. D. (2010). The sentient self. Brain Struct. Funct. 214, 563-577. doi: 10.1007/s00429-010-0248-y

Craig, A. D. (2015). How Do You Feel? An Interoceptive Moment with Your Neurobiological Self. Princeton, NJ: Princeton University Press.

Damasio, A. (2010). Self Comes to Mind: Constructing the Conscious Brain. New York, NY: Pantheon.

de Tommaso, M., Calabrese, R., Vecchio, E., De Vito Francesco, V., Lancioni, G., and Livrea, P. (2009). Effects of affective pictures on pain sensitivity and cortical responses induced by laser stimuli in healthy subjects and migraine patients. Int. J. Psychophysiol. 74, 139-148. doi: 10.1016/j.ijpsycho.2009. 08.004

Desmedt, O., Corneille, O., Luminet, O., Murphy, J., Bird, G., and Maurage, P. (2020). Contribution of time estimation and knowledge to heartbeat counting task performance under original and adapted instructions. Biol. Psychol. 154:107904. doi: 10.1016/j.biopsycho.2020.107904

Desmedt, O., Luminet, O., and Corneille, O. (2018). The heartbeat counting task largely involves non-interoceptive processes: evidence from both the original and an adapted counting task. Biol. Psychol. 138, 185-188. doi: 10.1016/j. biopsycho.2018.09.004

Di Lernia, D., Serino, S., and Riva, G. (2016). Pain in the body. altered interoception in chronic pain conditions: a systematic review. Neurosci. Biobehav. Rev. 71, 328-341. doi: 10.1016/j.neubiorev.2016.09.015

Dienes, Z. (2011). Bayesian versus orthodox statistics: which side are you on? Perspect. Psychol. Sci. 6, 274-290. doi: 10.1177/1745691611406920

Faul, F., Erdfelder, E., Lang, A.-G., and Buchner, A. (2007). G*Power 3: a flexible statistical power analysis program for the social, behavioral, and biomedical sciences. Behav. Res. Methods 39, 175-191.

Ferentzi, E., Bogdány, T., Szabolcs, Z., Csala, B., Horváth, Á, and Köteles, F. (2018). Multichannel investigation of interoception: sensitivity is not a generalizable feature. Front. Hum. Neurosci. 12:223. doi: 10.3389/fnhum.2018.00223

Ferentzi, E., Köteles, F., Csala, B., Drew, R., Tihanyi, B. T., Pulay-Kottlár, G., et al. (2017). What makes sense in our body? Personality and sensory correlates of body awareness and somatosensory amplification. Pers. Individ. Dif. 104, 75-81. doi: 10.1016/j.paid.2016.07.034

Fillingim, R. B., King, C. D., Ribeiro-Dasilva, M. C., Rahim-Williams, B., and Riley, J. L. (2009). Sex, gender, and pain: a review of recent clinical and experimental findings. J. Pain 10, 447-485. doi: 10.1016/j.jpain.2008.12.001

Füstös, J., Gramann, K., Herbert, B. M., and Pollatos, O. (2013). On the embodiment of emotion regulation: interoceptive awareness facilitates reappraisal. Soc. Cogn. Affect. Neurosci. 8, 911-917. doi: 10.1093/scan/nss089

Garfinkel, S. N., Seth, A. K., Barrett, A. B., Suzuki, K., and Critchley, H. D. (2015). Knowing your own heart: distinguishing interoceptive accuracy from interoceptive awareness. Biol. Psychol. 104, 65-74. doi: 10.1016/j.biopsycho. 2014.11.004

Goodin, B. R., Quinn, N. B., King, C. D., Page, G. G., Haythornthwaite, J. A., Edwards, R. R., et al. (2012). Salivary cortisol and soluble tumor necrosis factor$\alpha$ receptor II responses to multiple experimental modalities of acute pain. Psychophysiology 49, 118-127. doi: 10.1111/j.1469-8986.2011.01280.x

Grabauskaité, A., Baranauskas, M., and Griškova-Bulanova, I. (2017). Interoception and gender: what aspects should we pay attention to? Conscious. Cogn. 48, 129-137. doi: 10.1016/j.concog.2016.11.002

Gracely, R. H. (1999). Pain measurement. Acta Anaesthesiol. Scand. 43, 897-908. doi: 10.1034/j.1399-6576.1999.430907.x

Harver, A., Katkin, E. S., and Bloch, E. (1993). Signal-detection outcomes on heartbeat and respiratory resistance detection tasks in male and female subjects. Psychophysiology 30, 223-230.

Haslam, D. R. (1967). Individual differences in pain threshold and level of arousal. Br. J. Psychol. 58, 139-142.

Herbert, B. M., Pollatos, O., Flor, H., Enck, P., and Schandry, R. (2010). Cardiac awareness and autonomic cardiac reactivity during emotional picture viewing and mental stress. Psychophysiology 47, 342-354. doi: 10.1111/j.1469-8986. 2009.00931.x

Herbert, B. M., Pollatos, O., and Schandry, R. (2007). Interoceptive sensitivity and emotion processing: an EEG study. Int. J. Psychophysiol. 65, 214-227. doi: 10.1016/j.ijpsycho.2007.04.007

Hockley, J. R. F., González-Cano, R., McMurray, S., Tejada-Giraldez, M. A., McGuire, C., Torres, A., et al. (2017). Visceral and somatic pain modalities reveal NaV1.7-independent visceral nociceptive pathways. J. Physiol. 595, 26612679. doi: 10.1113/JP272837

James, W. (1884). What is an emotion? Mind 9, 188-205.

Jarosz, A., and Wiley, J. (2014). What are the odds? a practical guide to computing and reporting Bayes factors. J. Probl. Solving 7:2. doi: 10.7771/1932-6246.1167

JASP Team (2019). JASP(Version 0.9.0.1) [Computer Software] (Version 0.9.0.1). Available online at: https://jasp-stats.org/ (accessed March 15, 2019).

Jeffreys, S. H. (1998). The Theory of Probability, 3rd Edn. Oxford: Oxford University Press.

Jones, G. E., and Hollandsworth, J. G. (1981). Heart rate discrimination before and after exercise-induced augmented cardiac activity. Psychophysiology 18, 252-257.

Jones, G. E., Jones, K. R., Rouse, C. H., Scott, D. M., and Caldwell, J. A. (1987). The effect of body position on the perception of cardiac sensations: an experiment and theoretical implications. Psychophysiology 24, 300-311. doi: 10.1111/j.14698986.1987.tb00300.x

Katkin, E. S. (1985). Blood, sweat, and tears: individual differences in autonomic self-perception. Psychophysiology 22, 125-137. doi: 10.1111/j.1469-8986.1985. tb01573.x

Katkin, E. S., Blascovich, J., and Goldband, S. (1981). Empirical assessment of visceral self-perception: individual and sex differences in the acquisition of heartbeat discrimination. J. Pers. Soc. Psychol. 40, 1095-1101. doi: 10.1037/ 0022-3514.40.6.1095

Khalsa, S. S., Rudrauf, D., Sandesara, C., Olshansky, B., and Tranel, D. (2009). Bolus isoproterenol infusions provide a reliable method for assessing interoceptive awareness. Int. J. Psychophysiol. 72, 34-45. doi: 10.1016/j.ijpsycho.2008.08.010

Koch, A., and Pollatos, O. (2014). Cardiac sensitivity in children: sex differences and its relationship to parameters of emotional processing. Psychophysiology 51, 932-941. doi: 10.1111/psyp.12233

Logan, H. L., Gedney, J. J., Sheffield, D., Xiang, Y., and Starrenburg, E. (2003). Stress influences the level of negative affectivity after forehead cold pressor pain. J. Pain 4, 520-529. doi: 10.1016/j.jpain.2003.09.001

Ludwick-Rosenthal, R., and Neufeld, R. W. (1985). Heart beat interoception: a study of individual differences. Int. J. Psychophysiol. 3, $57-65$.

Lynn, R., and Eysenck, H. J. (1961). Tolerance for pain, extraversion, and neuroticism. Percept. Mot. Skills 12, 161-162. doi: 10.2466/pms.1961.12.2.161

Meagher, M. W., Arnau, R. C., and Rhudy, J. L. (2001). Pain and emotion: effects of affective picture modulation. Psychosom. Med. 63, 79-90. doi: 10.1097/ 00006842-200101000-00010

Merskey, H., and Bogduk, N. (eds) (1994). Classification of Chronic Pain, 2nd Edn. Available online at: http://www.iasp-pain.org/PublicationsNews/Content.aspx? ItemNumber=1673 (accessed March 15, 2019). 
Montgomery, W. A., Jones, G. E., and Hollandsworth, J. G. (1984). The effects of physical fitness and exercise on cardiac awareness. Biol. Psychol. 18, 11-22. doi: 10.1016/0301-0511(84)90022-X

Montoya, P., Schandry, R., and Müller, A. (1993). Heartbeat evoked potentials (HEP): topography and influence of cardiac awareness and focus of attention. Electroencephalogr. Clin. Neurophysiol. 88, 163-172.

Moseley, G. L. (2007). Reconceptualising pain according to modern pain science. Phys. Ther. Rev. 12, 169-178. doi: 10.1179/108331907X223010

Murphy, J., Brewer, R., Hobson, H., Catmur, C., and Bird, G. (2018). Is alexithymia characterised by impaired interoception? Further evidence, the importance of control variables, and the problems with the heartbeat counting task. Biol. Psychol. 136, 189-197. doi: 10.1016/j.biopsycho.2018.05.010

Mussgay, L., Klinkenberg, N., and Rüddel, H. (1999). Heart beat perception in patients with depressive, somatoform, and personality disorders. J. Psychophysiol. 13, 27-36. doi: 10.1027//0269-8803.13.1.27

Ostrom, C., Bair, E., Maixner, W., Dubner, R., Fillingim, R. B., Ohrbach, R., et al. (2017). Demographic predictors of pain sensitivity: results from the OPPERA study. J. Pain 18, 295-307.

Pennebaker, J. W. (1982). The Psychology of Physical Symptoms. New York, NY: Springer.

Pennebaker, J. W., and Roberts, T.-A. (1992). Toward a his and hers theory of emotion: gender differences in visceral perception. J. Soc. Clin. Psychol. 11, 199-212. doi: 10.1521/jscp.1992.11.3.199

Pollatos, O., Füstös, J., and Critchley, H. D. (2012). On the generalised embodiment of pain: how interoceptive sensitivity modulates cutaneous pain perception. Pain 153, 1680-1686. doi: 10.1016/j.pain.2012.04.030

Pollatos, O., Herbert, B. M., Matthias, E., and Schandry, R. (2007a). Heart rate response after emotional picture presentation is modulated by interoceptive awareness. Int. J. Psychophysiol. 63, 117-124. doi: 10.1016/j.ijpsycho.2006.09. 003

Pollatos, O., and Schandry, R. (2004). Accuracy of heartbeat perception is reflected in the amplitude of the heartbeat-evoked brain potential. Psychophysiology 41, 476-482. doi: 10.111/1469-8986.2004.00170.x

Pollatos, O., Traut-Mattausch, E., Schroeder, H., and Schandry, R. (2007b). Interoceptive awareness mediates the relationship between anxiety and the intensity of unpleasant feelings. J. Anxiety Disord. 21, 931-943. doi: 10.1016/ j.janxdis.2006.12.004

Rainville, P., Bao, Q. V. H., and Chrétien, P. (2005). Pain-related emotions modulate experimental pain perception and autonomic responses. Pain 118, 306-318. doi: 10.1016/j.pain.2005.08.022

Rhudy, J. L., and Williams, A. E. (2005). Gender differences in pain: do emotions play a role? Gend. Med. 2, 208-226.

Ring, C., and Brener, J. (2018). Heartbeat counting is unrelated to heartbeat detection: a comparison of methods to quantify interoception. Psychophysiology 55:e13084. doi: 10.1111/psyp.13084

Ring, C., Brener, J., Knapp, K., and Mailloux, J. (2015). Effects of heartbeat feedback on beliefs about heart rate and heartbeat counting: a cautionary tale about interoceptive awareness. Biol. Psychol. 104, 193-198. doi: 10.1016/j.biopsycho. 2014.12.010

Roberts, B. W. (2009). Back to the future: personality and assessment and personality development. J. Res. Pers. 43, 137-145. doi: 10.1016/j.jrp.2008.12. 015
Rouse, C. H., Jones, G. E., and Jones, K. R. (1988). The effect of body composition and gender on cardiac awareness. Psychophysiology 25, 400-407.

Schandry, R. (1981). Heart beat perception and emotional experience. Psychophysiology 18, 483-488. doi: 10.1111/j.1469-8986.1981.tb02486.x

Schandry, R., Bestler, M., and Montoya, P. (1993). On the relation between cardiodynamics and heartbeat perception. Psychophysiology 30, 467-474. doi: 10.1111/j.1469-8986.1993.tb02070.x

Svensson, P., Bjerring, P., Arendt-Nielsen, L., Nielsen, J. C., and Kaaber, S. (1991). Comparison of four laser types for experimental pain stimulation on oral mucosa and hairy skin. Lasers Surg. Med. 11, 313-324. doi: 10.1002/lsm. 1900110402

Villemure, C. (2002). Cognitive modulation of pain: how do attention and emotion influence pain processing? Pain 95, 195-199.

Villemure, C., Slotnick, B. M., and Bushnell, M. C. (2003). Effects of odors on pain perception: deciphering the roles of emotion and attention. Pain 106, 101-108.

Watson, D., Clark, L. A., McIntyre, C. W., and Hamaker, S. (1992). Affect, personality, and social activity. J. Pers. Soc. Psychol. 63, 1011-1025. doi: 10.1037/ 0022-3514.63.6.1011

Watson, D., Clark, L. A., and Tellegen, A. (1988). Development and validation of brief measures of positive and negative affect: the PANAS scales. J. Pers. Soc. Psychol. 54, 1063-1070. doi: 10.1037/0022-3514.54.6.1063

Weiss, S., Sack, M., Henningsen, P., and Pollatos, O. (2014). On the interaction of self-regulation, interoception and pain perception. Psychopathology 47, 377382. doi: $10.1159 / 000365107$

Werner, N. S., Duschek, S., Mattern, M., and Schandry, R. (2009). The relationship between pain perception and interoception. J. Psychophysiol. 23, 35-42. doi: 10.1027/0269-8803.23.1.35

Whitehead, W. E., Drescher, V. M., Heiman, P., and Blackwell, B. (1977). Relation of heart rate control to heartbeat perception. Biofeedback Self Regul. 2, 371-392. doi: 10.1007/BF00998623

Zamariola, G., Maurage, P., Luminet, O., and Corneille, O. (2018). Interoceptive accuracy scores from the heartbeat counting task are problematic: evidence from simple bivariate correlations. Biol. Psychol. 137, 12-17. doi: 10.1016/j. biopsycho.2018.06.006

Zautra, A. J., Johnson, L. M., and Davis, M. C. (2005). Positive affect as a source of resilience for women in chronic pain. J. Consult. Clin. Psychol. 73, 212-220. doi: 10.1037/0022-006X.73.2.212

Zimprich, D., Nusser, L., and Pollatos, O. (2020). Are interoceptive accuracy scores from the heartbeat counting task problematic? A comment on Zamariola et al. (2018). Biol. Psychol. 152:107868. doi: 10.1016/j.biopsycho.2020.107868

Conflict of Interest: The authors declare that the research was conducted in the absence of any commercial or financial relationships that could be construed as a potential conflict of interest.

Copyright (C) 2021 Ferentzi, Geiger, Mai-Lippold, Köteles, Montag and Pollatos. This is an open-access article distributed under the terms of the Creative Commons Attribution License (CC BY). The use, distribution or reproduction in other forums is permitted, provided the original author(s) and the copyright owner(s) are credited and that the original publication in this journal is cited, in accordance with accepted academic practice. No use, distribution or reproduction is permitted which does not comply with these terms. 